\title{
The influence of MTHFR risk knowledge on changes in folate intake: results from the Food4Me study
}

\author{
C.B. O’Donovan ${ }^{1}$, M.C. Walsh ${ }^{1}$, C. Celis-Morales ${ }^{2}$, J. Bouwman ${ }^{3}$, K.A. Grimaldi ${ }^{4}$, \\ C.A. Devon ${ }^{5}$, Y. Manios ${ }^{6}$, I. Traczyk ${ }^{7}$, A. Martinez ${ }^{8}$, W. Saris ${ }^{9}$, H. Daniel ${ }^{10}$, J. Lovegrove ${ }^{11}$, \\ J.C. Mathers, M.J. Gibney ${ }^{1}$, L. Brennan ${ }^{1}$ and E.R. Gibney ${ }^{1}$ on behalf of the Food4Me study \\ ${ }_{1}^{1}$ Institute of Food \& Health, University College Dublin, Dublin 4, Ireland, ${ }^{2}$ Human Nutrition Research Centre, Institute \\ of Cellular Medicine, Newcastle University, Newcastle, NE4 5PL, UK, ${ }^{3}$ TNO, Microbiology and Systems Biology \\ Group, Zeist, the Netherlands, ${ }^{4}$ Eurogenetica Ltd, 7 Salisbury Road, Burnham-on-Sea, TA8 1HX, UK, ${ }^{5}$ Department of \\ Nutrition, Institute of Basic Medical Sciences, University of Oslo, Oslo, Norway, ${ }^{6}$ Department of Nutrition and \\ Dietetics, Harokopio University, Athens, Greece, ${ }^{7}$ National Food \& Nutrition Institute, Warsaw, Poland, ${ }^{8}$ Department \\ of Nutrition, Food Science and Physiology, University of Navarra, Pamplona, Spain, ${ }^{9}$ Department of Human Biology, \\ NUTRIM, Maastricht University, Maastricht, The Netherlands, ${ }^{10}$ ZIEL Research Center of Nutrition and Food \\ Sciences, Biochemistry Unit, Technische Universität München, Munich, Germany and ${ }^{11}$ Hugh Sinclair Unit of Human \\ Nutrition and Institute for Cardiovascular and Metabolic Health, University of Reading, Reading, RG6 6AR, UK
}

The methylenetetrahydrofolate reductase (MTHFR) gene plays a key role in folate metabolism. Evidence suggests that adequate folate status is important for lowering the risk of heart disease and stroke through its involvement in homocysteine recycling ${ }^{(1)}$. Therefore it is essential for those with the risk variants of MTHFR (CT \& TT genotypes) to have adequate folate intakes. Recent evidence supports the view that disclosure of genetic risk can influence dietary and lifestyle modifications ${ }^{(2)}$. The objective of this study was to examine whether knowledge of MTHFR risk would improve folate intake more in individuals carrying the risk variants (CT, TT genotypes at codon 677) compared to those with the non-risk variant (CC genotype).

To address this objective, data was analysed from the Food4Me Proof-of-Principle (PoP) study. Participants were recruited across seven centres throughout Europe $(n=1,607)$ to take part in this six month internet based personalised nutrition intervention study, where they received varying levels of personalised nutrition advice ${ }^{(3)}$. The current study used data from those participants randomised to receive feedback based on diet, phenotype and genotype. Information was provided on five diet-related genes including MTHFR $(n=332)$. Individuals were informed whether they had the risk or non-risk versions of the gene in relation to cardiovascular health and importance of sufficient dietary folate intake. For analysis purposes, participants were split into risk $(\mathrm{n}=187)$ and non-risk groups $(n=145)$ and differences between these groups analysed. Independent t-tests were performed to investigate baseline characteristics. Data was logged where necessary. Paired t-tests were employed to investigate changes in folate intake between baseline and month 3 for each of the groups. General linear models were used to assess folate intake differences between the groups.

The mean ages of the risk and non-risk groups were $41 \pm 13$ years and $40 \pm 14$ years respectively. Both groups were slightly overweight with mean BMI $25.45 \pm 4.85 \mathrm{~kg} / \mathrm{m}^{2}$ for the risk group and $25.96 \pm 4.91 \mathrm{~kg} / \mathrm{m}^{2}$ for the non-risk group. There were no differences at baseline between the groups for any characteristics including age, gender and BMI. Baseline folate intakes for the risk and non-risk groups were $408 \pm 169$ and $393 \pm 190 \mathrm{mg}$ per $10 \mathrm{MJ}$ energy respectively. Both groups increased their folate intake at month 3 with intakes of $431 \pm 223$ and $412 \pm 183 \mathrm{mg}$ per $10 \mathrm{MJ}$ energy for the risk and non-risk groups respectively. There was no significant difference between the groups for changes in folate intakes at month 3 compared with baseline. There were also no differences between genotype-based risk groups for changes in the intake of folate-containing food groups including green leafy vegetables, beans \& legumes and folate-fortified cereals at month 3 compared with baseline. This work suggests that knowledge of MTHFR risk does not improve folate intake more in individuals with the risk variant compared to those with the non-risk variant. Future work is warranted to confirm these findings for improving personalised nutrition practices.

The work was funded by Food4Me (KBBE.2010·2·3-02, Project no. 265494.

1. Wang X, Qin X, Demirtas H, Li J et al. (2007) Lancet 369, 1876-1882.

2. Nielsen DE \& El-Sohemy A (2014) PLOS ONE 9, e112665.

3. Celis-Morales C, Livingstone KM, Marsaux CFM et al. (2015) DOI: 10.1007/s12263-014-0450-2 